\title{
CONSIDERATIONS ON ACTIVE CONTROL OF CROSSWIND STABILITY OF RAILWAY VEHICLES ${ }^{1}$
}

\author{
Andreas Heckmann $^{\text {a2 }}$, Bernhard Kurzeck ${ }^{a}$, Tilman Bünte ${ }^{a}$, Sigfried Loose \\ ${ }^{a}$ Institute of System Dynamics and Control, \\ DLR German Aerospace Center, P.O.Box 1116, 82230 Wessling, Germany \\ ${ }^{\mathrm{b}}$ Institute of Aerodynamics and Flow Technology \\ DLR German Aerospace Center, Bunsenstr. 10, 37073 Göttingen, Germany
}

\begin{abstract}
The DLR research project Next Generation Train deals with concepts, methods and technologies for a very high-speed train in double deck configuration and light-weight design. Due to these three key features crosswind stability is a particular subject of study. It is shown that conventional approaches here fall short of guaranteeing safety in high-wind occurrences according to the given homologation standards. Therefore this paper discusses the feasibility of different approaches to ensure crosswind stability by means of active control. Four different concepts are overviewed, the most promising one is then chosen und examined in detailed multibody simulations that are based on data from wind tunnel measurements of the Next Generation Train.
\end{abstract}

Keywords: crosswind stability, railway vehicle, multibody simulation, vehicle control

\section{INTRODUCTION}

Crosswind stability is a crucial safety issue of high-speed trains and gains additional importance with every step the maximum vehicle speed in operation is increased [1]. Consequently standards have been established in Europe that define evaluation procedures and limits to be met in order to homologate a new vehicle type [2, 3].

Hence, these approval criteria are a stringent requirement in train design, in which two main approaches to comply with are known: train designers may optimize either the outer geometry of the train with respect to crosswind sensitivity [4] or the weight and the weight distribution of the train in order to find beneficial configurations [5]. These approaches will stay important in the future. However, it has to be stated that the potential of these methods to provide relevant solutions are limited by the laws of physics and by significant design conflicts. For example, the vehicle weight may only be increased up to a certain limit since the maximum wheel-rail forces are another homologation criterion to be met.

This general background provides the motivation of a number of associated activities within the DLR project Next Generation Train (NGT) that concerns three particular key features: a very high speed train (400 km/h maximum speed in operation) in double deck configuration and light-weight design [6], see Figure 1. It is obvious that these three key issues together imply a major challenge with respect to crosswind stability.

The associated activities of the NGT certainly include the above-mentioned conventional approaches, i.e. deal with aerodynamically beneficial shapes and advantageous weight distributions. In addition new experimental facilities were established that help to advance the fundamental knowledge on the characteristics of the flow field around the vehicle e.g. in transient conditions, see [7]. Furthermore, Carrarini [8] proposed the application of a reliability based analysis of the crosswind stability problem in order to more efficiently assess the risk and to

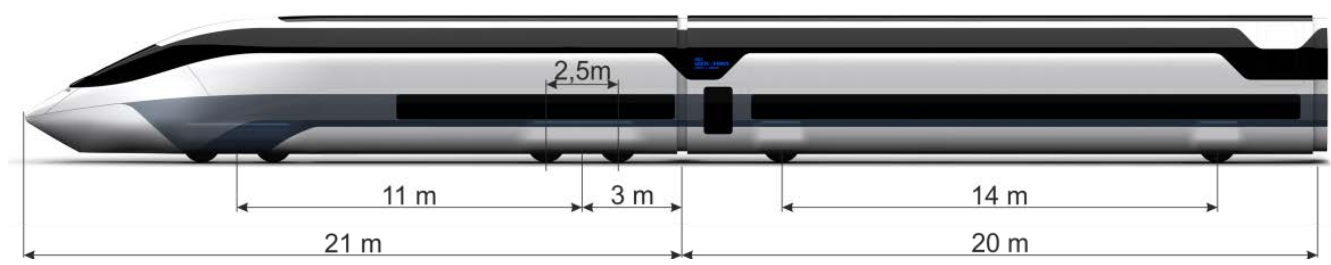

Figure 1: Illustration of the NGT train concept with train head and first coach [6, p. 10]

\footnotetext{
${ }^{1}$ This is an Authors' Accepted Manuscript of an article published in Vehicle System Dynamics : International Journal of Vehicle Mechanics and Mobility, 52:6, 759-775, 2014, DOI: 10.1080/00423114.2014.901539.

${ }^{2}$ Corresponding author: Tel.: +49 8153 281699, Email: andreas.heckmann@dlr.de
} 
reduce the safety margins that are included in today's homologation rules.

However, the focus of this paper is rather the following topic: Does active control in principle provide options to cope with the crosswind stability problem and what may promising control concepts look like? In order to answer these questions the paper will discuss the following four approaches:

1. Suspension Control (SC): Orvnäs et al. [9] present an active control of the lateral secondary suspension in order to improve ride comfort but also shortly mention benefits for crosswind stability without further comments. In fact the lateral displacement and the swaying motion of the carbody enabled by the secondary suspension influence the instantaneous position of the center of gravity and in turn effect the wheel unloading.

2. Weight Shifting (WS): Besides suspension control Carrarini [10] also examines the idea to temporarily shift heavy underbelly equipment like transformers and adjust the weight distribution in such a way that crosswind stability is improved e.g. by active load displacement towards the front bogie.

3. Aerodynamic Downforce (AD): An additional loading of those running gears that are prone for crosswind induced wheel unloading could also be achieved by aerodynamic control surfaces that generate forces that are directed downwards.

4. Attraction Control (ACO): Hofmann [11], Werle [12] and Funieru [13] studied the design of so called boosters, i.e. electromagnetic actuators that induce additional attracting and tangential (accelerating or braking) forces between the bogie and the rail. Their primary scope of work was the temporary improvement of the traction behavior, but in principle these actuators could also be utilized in order to counteract the wheel unloading due to crosswinds.

As already indicated by the references given above, this paper is not the first one that addresses active control of crosswind stability. However in all referred cases it has been treated as a minor or side issue out of the actual focus of the authors. This is different for the present paper. Due to the particularities of the NGT project the crosswind stability problem becomes a matter of special importance. This fact is demonstrated with evaluations based on wind tunnel measurements of the NGT in Section 2 that provide a specific background for further discussion. The four approaches introduced above are then reviewed in Section 3 in more details. The Attraction Control approach is extensively examined in Section 4. Finally Section 5 concludes the results of the preceding considerations.

\section{Detailed Problem Statement}

From the very beginning of the NGT project, it has been clear that a very high speed train in double-deck configuration and light weight design is a very demanding concept in particular regarding crosswind stability. This challenge has been set up by intention since it specifies to what end the research on the assessment methodology and the development of promising solution approaches in this field may be good for.

As soon as the NGT train design had reached a certain maturity and proposals for the outer train geometry, the mass distribution, the stiffness and damping properties of the primary and secondary suspensions were available, a survey on the crosswind behavior of the NGT was launched. Figure 2 combines the presentation of the experimental set-up of wind tunnel measurements with the animation of the simulated flow and pressure field of the NGT. As a result of these experimental efforts the aerodynamic coefficients for the NGT train head and the first

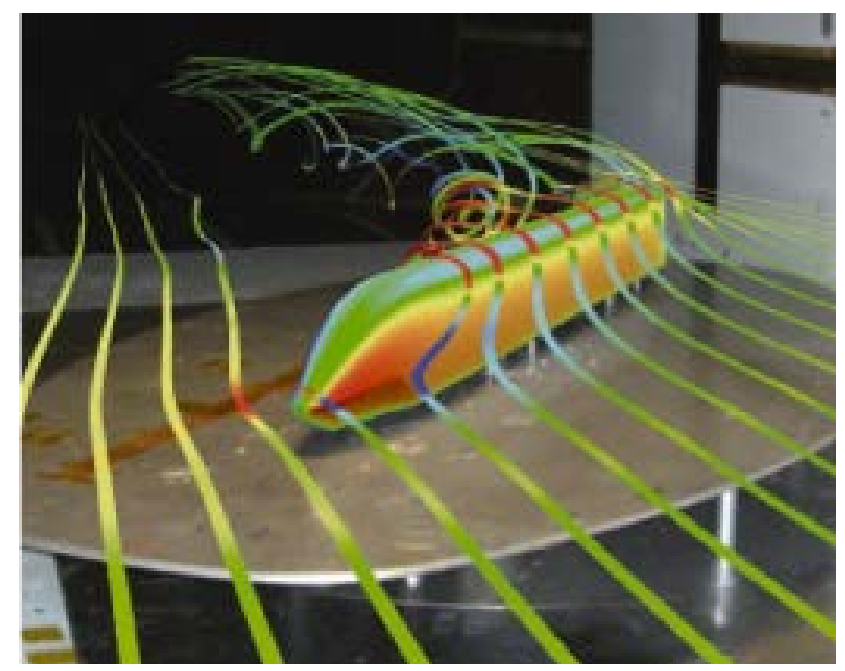

Figure 2: Calculated streamlines and pressure distribution on the NGT model scale 1:25 in the Cologne Cryogenic Wind Tunnel under cross wind conditions 


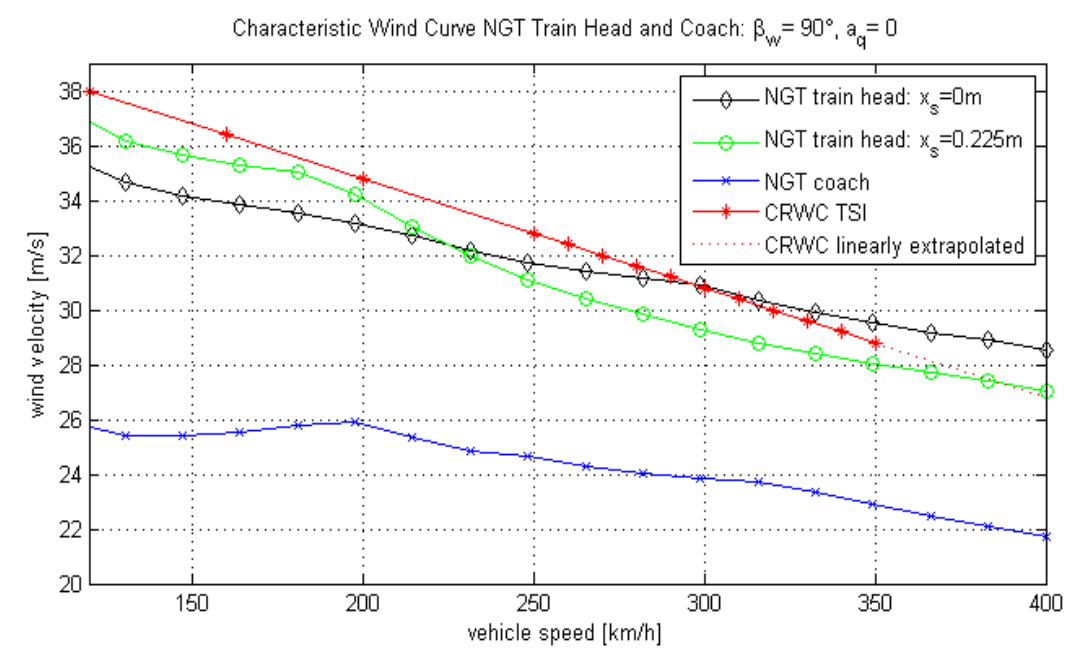

Figure 3: Characteristic Wind Curve of the NGT train head with two different weight distributions and the NGT coach compared to the reference curve CRWC from TSI

intermediate car or the second car of the train set, respectively, were obtained. In the next step in particular the results associated to the running on flat ground scenario according to RIL 807 [14] with a Reynolds Number of $0.75 \cdot 10^{6}$ were utilized in multibody simulations of the NGT train as described in the sequel.

For this purpose a software tool called Crosswind Stability based on the modeling language Modelica [15] was implemented and validated by means of the two reference data sets provided with the EN14067 [3]. The basis scenario is defined as follows: At given train speed, wind velocity and wind direction quasi-static forces and torques calculated using the above-mentioned aerodynamic coefficients are applied to a simplified multibody train model. These applied forces and torques lead to an unloading of the wheels at the windward side of the train. According to the EN14067-6 the so-called critical wind speed is defined as the wind velocity, at which the remaining wheel load is $10 \%$ of the static load. The critical wind speed is evaluated for several train velocities, these sample points are then connected to construct a curve called the Characteristic Wind Curve (CWC). In order to meet the homologation criterion the CWC of the considered vehicle must completely run above the Characteristic Reference Wind Curve (CRWC) defined by the TSI [2], see Figure 3.

The NGT train head is assembled with heavy traction equipment while the storage of luggage separated from the passenger areas is its only intended payload. In the initial least weight but ready to run configuration that is represented by the black curve in Figure 3, each of the eight wheels, mounted as double wheel pairs in two running gears carries a static wheel load of $74.6 \mathrm{kN}$. However the optimization procedure showed that an uneven load distribution performs better with respect to crosswind stability. The center of gravity of the carbody should be displaced by $0.225 \mathrm{~m}$ from the central position towards the leading running gear. In consequence, the static wheel load of the empty train head increases at the leading running gear up to $78.6 \mathrm{kN}$ and decreases to $70.6 \mathrm{kN}$ at the trailing running gear. The green curve in Figure 3 presents the resulting CWC of the NGT train head. Due to the optimization the CWC approximately parallels to the CRWC. Therefore, the situation is improved for vehicle speeds below $225 \mathrm{~km} / \mathrm{h}$, but worsened for higher velocities. With or without weight shift, the CWC runs below the CRWC for major velocities ranges, i.e. the NGT performs worse than requestedHence, the NGT train head could not be homologated according to the TSI.

The situation is even worse for the intermediate NGT coaches that run on a total of four wheels each with $58 \mathrm{kN}$ static wheel load in the least weight but ready to run configuration. While the critical wind speed is just 1 to $2 \mathrm{~m} / \mathrm{s}$ below the CRWC for the train head, the intermediate coaches fail to meet the homologation criterion by about 12 $\mathrm{m} / \mathrm{s}$. So, there does not seem to be any conventional option to find a remedy. The two car types perform that much different, since the coach has less than half the weight of the train head, only two wheel pairs instead of four, but nearly the same length according to Figure 1 . This result clearly confirms the initial assumption: the crosswind stability of such train configuration is beyond today's feasibility.

\section{GENERAL DISCUSSION OF THE SOLUTION APPROACHES}

\subsection{Suspension Control}

It is the task of the secondary suspensions to decouple the motion of the carbody from the dynamics of the running gears. This is achieved by comparable low stiffness parameters. However, as soon as significant lateral wind forces are applied, these low stiffnesses enable the carbody to move out of the central position, the wind torques excite a 


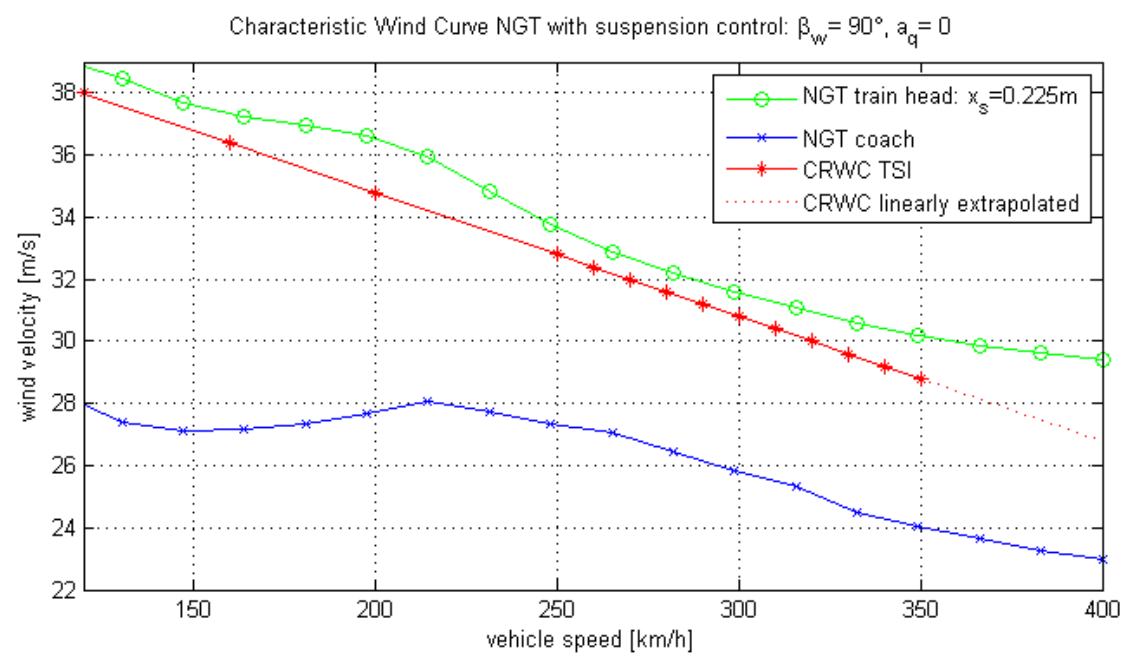

Figure 4: Potential improvement of CWCof the NGT by Suspension Control

rolling motion of the carbody. Both motion components lead to a significant displacement of the center of gravity of the carbody and amplify the unloading of the wheels at the windward side. Additionally, an angular deflection of the carbody induces higher rolling moments which is a flow field feed-back similar to aeroelastic effects in aircraft aerodynamics [16], [17]. As a consequence in today's train design, bump stops are introduced that limit the maximum lateral displacements and rolling motion according to crosswind stability requirements, see [18] concerning the stabilizing effect of reduced bump stop clearances.

There are two options to improve this behavior by manipulation of the secondary suspensions:

a) The stiffness properties in lateral direction may be adjusted by feed-forward control action as soon as high crosswinds are expected. This will of course temporarily reduce the ride comfort of the passengers but helps to reduce the lateral deflection as well.

b) A feedback controller of the lateral suspension may be specified in such a way that the lateral displacement is reduced as quickly as possible and the lateral and rolling motion is limited in narrow margins. This concept could as well include adaptive feed-forward control in order to adapt to weather forecast information, track-side and vehicle-side measurements on crosswinds, see Section 4.2.

To a certain extent the design of such a system would show significant similarities to already implemented systems e.g. [19] that control the rolling motion in curves to allow for higher train speeds.

In order to estimate the potential of Suspension Control with respect to the CWC of the NGT, a Crosswind Stability analysis was performed as described in Section 2, but the lateral suspensions were stiffened by three orders of magnitude. Clearly in reality, this set-up would result in inacceptable running and comfort properties, however here it was intended to quickly provide upper bounds for the potential improvements one might expect by suspension control. Figure 4 presents the two CWC plots for the NGT train head and the coach. In general the critical wind speeds could be shifted up for about $2 \mathrm{~m} / \mathrm{s}$ with this measure compared to the results from Figure 3 . The CWC of the NGT train head is thereby raised above the CRWC, so in principle the chances for homologation of the train head were advanced. But so far, there is no solution apparent for the coach.

\subsection{Weight Shifting}

Carrarini [10] proposed to actively move heavy underbelly equipment such as transformers in order to influence the mass distribution. That way the stability properties may be improved taking the actual load distribution and current running speed into account and introducing additional options compared to an optimized but fixed weight distribution. Already the comparison of the NGT train curves with $x_{s}=0 m$ and $x_{s}=0.225 m$ in Figure 3 indicates that a specific weight distribution might be beneficial for one vehicle speed region but harmfull for other regions. This characteristics could be exploited if the weight distribution can be adapted to the vehicle velocity.

The idea was inspired by Johannsen et al. [20], who suggested an active vibration control system that moves underbelly masses in the lateral direction. In theory a lateral motion could be also very efficient to counteract wheel unloading. However, every measure that assumes the wind direction and is laterally unsymmetric is prone to changing winds, vortex or orographic effects and induce a high additional failure risk. Therefore Carrarini considered a feed-forward control of the longitudinal position of heavy equipment based on up-to-date weather information or track-side measurements. A drawback is that a temporary increase of the wheel-rail forces beyond the conventionally admissible limit has to be accepted. A study on the potential showed that the possible 


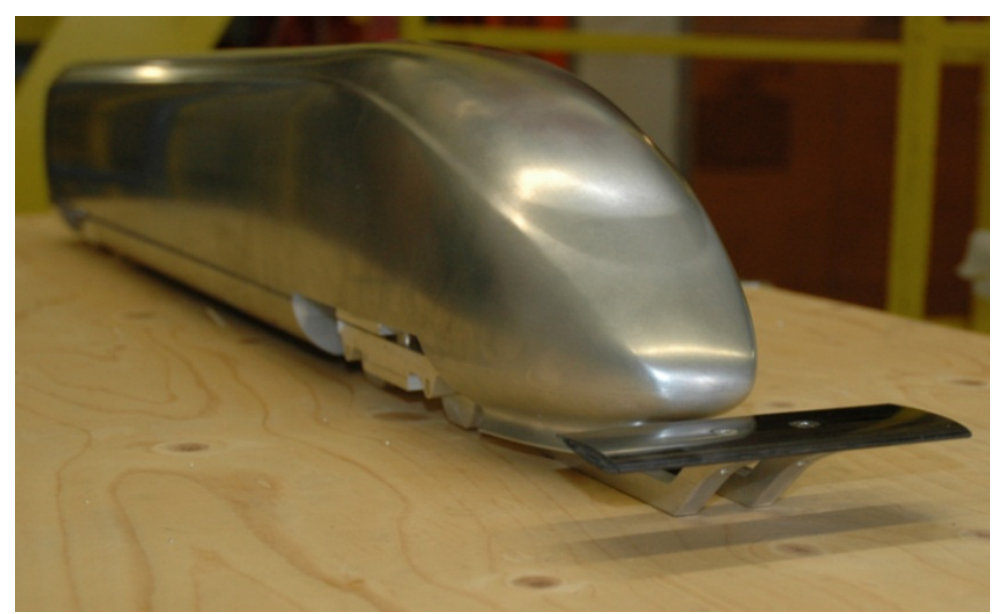

Figure 5: Wind tunnel model for the examination of fundamental aerodynamic and aeroacoustic properties of a front spolie $r$ [22]

improvements with respect the CWC are in the same order of magnitude as it was shown for the suspension control concept above. However, the concept is more or less specifically suited for the train head and the additional loading of its leading running gear in the case of available warning information. Though, the problem of the coaches is rather too little load at both running gears and not just at one of them. This is why this concept was not followed up within the NGT project.

\subsection{Aerodynamic Downforce}

In preliminary brainstorming discussions in the NGT team the question emerged whether the aerodynamic characteristics of the train could be influenced in such a way that a downforce could be generated as it is done for instance for Formula One racing cars [21] In principle such a downforce could counteract the wheel unloading and increase the safety margin under sidewind conditions. A dynamic analysis yielded that a maximum of $23 \mathrm{kN}$ aerodynamic force is required to lift the CWC of the train head to meet the CRWC. Therefor he wheel/rail load could be kept below the 8 to limit due to the movement of the center of gravity of about $0.27 \mathrm{~m}$ towards the trailing running gear. Based on a comparison to rear wings of race cars [21] the required wing area to generate $23 \mathrm{kN}$ downforce at $250 \mathrm{~km} / \mathrm{h}$ was estimated to $2.6 \mathrm{~m}^{2}$. Lower speeds demand for larger wing areas while smaller wings are sufficient to generate the same downforce at higher speeds. These relations in principle seem to be feasible. However, typical lift-to-drag ratios of such configurations are not higher than 3 , so that an additional drag force of about $8 \mathrm{kN}$ is to be expected. Wind tunnel measurements with a scaled model of an ICE 3 train head [22] that was equipped with a front spoiler, see Figure 5, confirmed a significant increase of the drag for train geometries as well., which of coursecontradicts the energy efficiency goals of the NGT.. In addition such a measure was assumed to be applicable to the NGT train head only, but not for intermediate coaches, so that aerodynamic control surfaces are no answer to the most relevant problem, which is the coach as presented in Figure 3.

\subsection{Attraction Control}

Hörl et al. [23] generally discussed application fields of the electromagnetic linear motor technology in the rail-wheel-system, but rejected the suitability of linear actuators for crosswind stability enhancement at that time. However research results obtained in the meantime, see [11], [12] and [13], offer a more precise view on feasible design variants of electromagnetic actuators for wheel-rail applications and their performance, although the activities are rather focused on traction effort than on crosswind stability. However, the general idea of applying an additional attracting force between running gear or wheel and rail can be exploited for both purposes.

As a first step to qualify the potential of this concept, another Crosswind Stability analysis was performed, but now additional forces at each running gear are introduced. These forces are defined in such a way that the requested critical wind speeds are exactly met. Consequently in this set-up, the CWC precisely matches the CRWC which

\begin{tabular}{|l|c|}
\hline Required additional attracting force to satisfy the wheel unloading criterion from EN 14067-6 \\
\hline NGT train head (w/o Suspenion Control) & $10 \mathrm{kN}$ \\
\hline NGT coach w/o Suspension Control & $47 \mathrm{kN}$ \\
\hline NGT coach with additional Suspension Control system & $39 \mathrm{kN}$ \\
\hline
\end{tabular}

Table 1: Table with required attracting forces at each running gear to ensure Crosswind Stability 


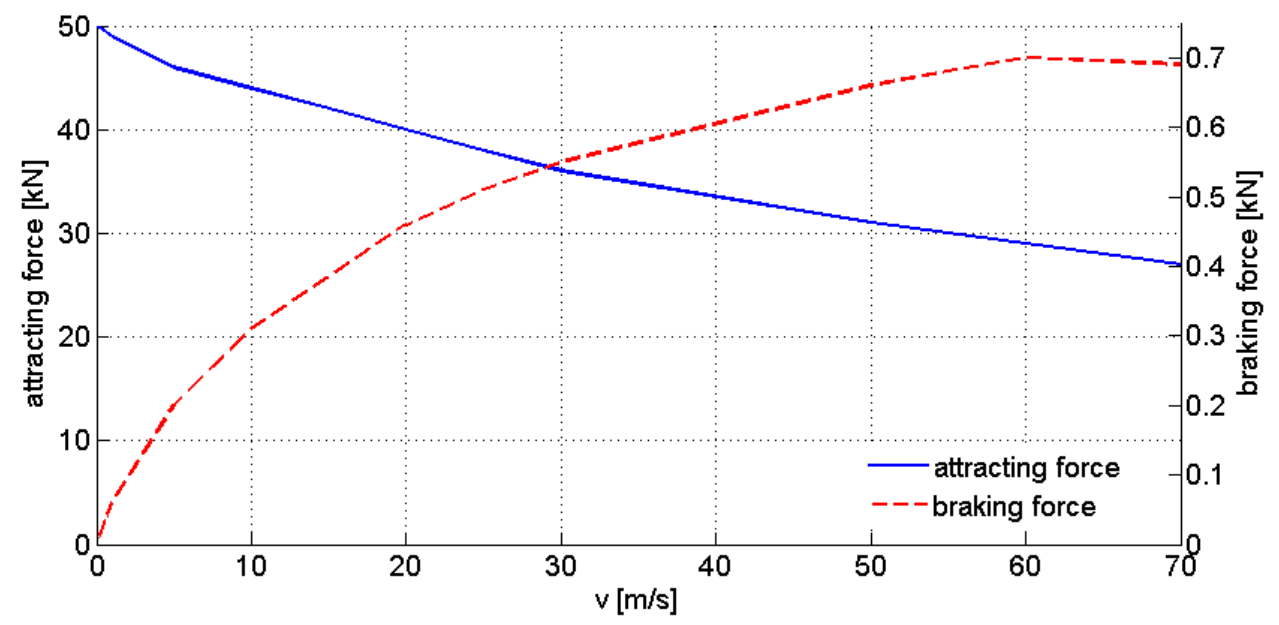

Figure 6: Calculated attracting and braking force of a DC linear booster of about $215 \mathrm{~kg}$ weight according to Werle [12, p. 100]

was linearly extrapolated up to the $400 \mathrm{~km} / \mathrm{h}$ for this purpose. Table 1 presents the maximum forces per running gear that were obtained that way. If $10 \mathrm{kN}$ additional attracting force is available at the windward side of each running gear of the NGT train head, the minimum wheel rail force takes on $10 \%$ of the static wheel-rail force. The corresponding value for the NGT coach reaches $47 \mathrm{kN}$. An additional analysis adapting the set-up as introduced in Sec. 3.1 showed that this value can be reduced to $39 \mathrm{kN}$, if the two concepts Attraction Control and Suspension Control are combined. Thus, the highest forces are required for vehicle speeds from 150 to $200 \mathrm{~km} / \mathrm{h}$. This fact might already be concluded from the curve progression of the CWC of the NGT coach in Figure 3.

Werle [12] evaluated the attracting force of his DC actuator pre-design as a function of the train speed in Figure 6. Furthermore he demonstrated that by means of secondary actuator control its dynamics can be represented by a first order time lag with a time constant of $30 \mathrm{~ms}$ or less. This had been demanded by Hörl et al. [23]. Funieru [13] optimized the lay-out and achieved $22 \%$ higher attracting forces than Werle, up to $62 \mathrm{kN}$, but investigated velocities up to $160 \mathrm{~km} / \mathrm{h}$ only. So finally and in particular in view of the comparison of Table 1with Figure 6, it may be concluded: it seems to be feasible to design a DC actuator in such a way, that the required attracting forces are generated. This resume motivates a more detailed case study in the next section.

\section{Detailed Survey on the AtTraction Control Approach}

\subsection{Electromagnetic Attraction Force Actuator (EMA)}

In order to give an impression what a feasible actuator design for crosswind stability purposes may look like, this subsection intensively refers to research activities at the Institute of Electrical Energy Conversion, Darmstadt University of Technology, as already done in Section 3.4 to study the general feasibility, see [11], [12] and [13]. These activities as well reviewed other actuator concepts such as the asynchronous linear machine, but finally favored the DC actuator due to its simplicity and efficiency.

An electromagnetic DC actuator lay-out for large attracting forces shows some similarities to eddy current brakes, e.g. see Figure 7: The actuator is attached to the running gear; in operation, it moves downwards leaving an air gap of about $5 \mathrm{~mm}$ to the rail being its secondary and the coils are excited by DC current. However, the separated coils in eddy current brakes are composed in such a way that south and north poles alternate in longitudinal direction along the rail, so that large eddy currents and in turn large braking forces are induced.

In order to optimize the actuator for large attracting but little braking forces, Werle [12] and later Funieru [13] proposed a transversal flux lay-out, i.e. the windings of the single coil are arranged in parallel to the rail, so that the two magnetic poles are positioned laterally to the rail head. The final design of Funieru [13] requires a power supply of $25 \mathrm{~kW}$, provides a maximum attracting force of $62 \mathrm{kN}$ and has the dimensions $1200 \cdot 228 \cdot 130 \mathrm{~mm}$.

In the case of traction effort improvement the braking force arising due to the relative motion between actuator and the rail, see Figure 6, is an undesired side effect which reduces the efficiency of the booster. However for crosswind stability, the actuator isassumed to be in operation in the case of emergency only and the braking side effect is not obstructive here. 


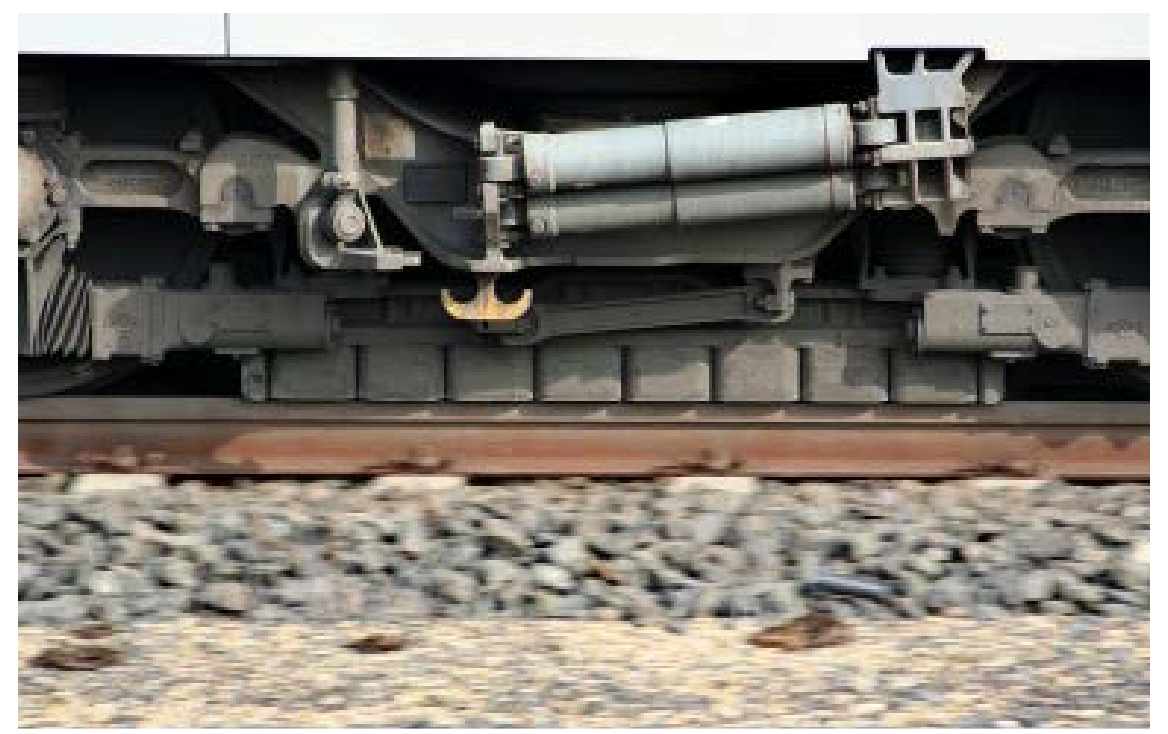

Figure 7: Linear eddy current brake of an ICE 3 in action [27]

An open point is the electromagnetic compatibility, since it turned out “... that the actuator is not compatible with the standard track sensors which are used in the German railway grid...” [13, p. 142] due to its high magnetic stray field.

There are some issues where the specific requirements of crosswind stability and the NGT train concept give reasons to reconsider the design of the EMA as presented above. Crosswind stability is a crucial safety issue so that rigorous reliability needs are given. Therefore it might be better not to use one single coil per actuator. If this single coil fails, the complete actuator fails which is to avoid by all means.

In addition the use of multiple coils would offer the capability of switching between different compositions of magnetic poles, e.g. one composition with alternating poles for braking purposes and one composition with uni-directional orientation for traction effort improvement and crosswind stability. Such dual use of the actuator reflects well the light-weight goals of the NGT project.

Another open point is the mounting of such an actuator at the single wheel pair running gears of the NGT coaches. In conventional bogies the actuator is mounted between the two wheels and the actuator in operation is symmetrically supported by the wheel bearings. That way the specified air gap between actuator and rail is guaranteed and no pitch motion is excited. The geometrical and mounting conditions are more difficult with a single wheel pair running gear where two actuators have to be mounted symmetrically on both sides of the wheel.

For traction effort improvement the attracting force of the actuator is part of a closed force circuit and deforms the rail between the two wheels only, see Section 4.5 in [12]. There is no resulting force on the rail directed upwards, that might pull the rail and the sleepers out of the subgrade. This is different for the crosswind stability application. According to Table 1,,the actuator at the NGT coach is supposed to apply $47 \mathrm{kN}$ to retain $10 \%$ of the static wheel load, which is $5.8 \mathrm{kN}$ according to Section 2. This means: a resulting force of $F=-41.2 \mathrm{kN}$ pulls the rail upwards, see Figure 8. This leads to a totally untypical, dynamical loading of the rail and its mounting and is much more than it could be counteracted by the weight of the rail including concrete sleepers (together ca. $530 \mathrm{~kg} / \mathrm{m}$ ).

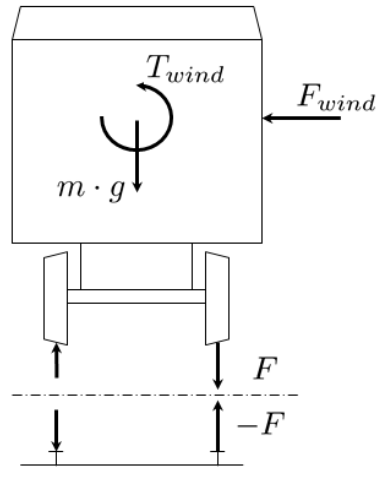

Figure 8: Schematic 2D-sketch to present the resulting force F that prevents the overturning of the vehicle but as well initiates the upward force acting on the rail. 
The ballast bed of conventional tracks is not designed to transmit forces that are directed upwards, so that Attraction Control seems to be applicable on slab track only.

\subsection{Sensor Concept}

The idea to improve crosswind stability by feedback control relies on sensor information of the current vertical force between wheel and rail which is the relevant measure to quantify the stability of the vehicle in the case of high crosswinds according to the EN 14067-6. In general, several measurement methods are possible, e.g. an observer that is based on the measurement of the vertical force in the primary suspension.

However, the NGT considers active control of the running dynamics as well. Fortunately, the associated control and sensor concept already provides sensor information that may also be exploited for active crosswind stability control. NGT has running gears with independently rotating wheels and two axle intermediate coaches with an axle distance of $14 \mathrm{~m}$. In order to ensure a good running performance in curves and on disturbed track with low wheel wear, active guiding of the wheel pairs is needed. For this mechatronic track guidance, the lateral displacement of the wheel pair is the required quantity to be measured or estimated.

Within the NGT project, a discussion on different measurement options led to the conclusion, see [24]: For the mechatronic track guidance in the NGT an indirect method is preferred using force and torque sensors in the mounting of the stub shaft of each wheel. The lateral displacement is estimated on the basis of the measured force and torque signals. This measurement concept also provides an estimation for the current vertical force in the rail/wheel interface that may be used for active crosswind stability control as presented in the succeeding section.

\subsection{Control Concept}

The control concept we suggest here as a draft is based on the assumption that the vertical wheel load force can be measured or estimated with sufficient precision (cf. Section 4.2) and that the EMA is powerful enough to provide sufficiently high forces (cf. Section 4.1). Nonlinear dynamic force feedback is used which will be described in the sequel.

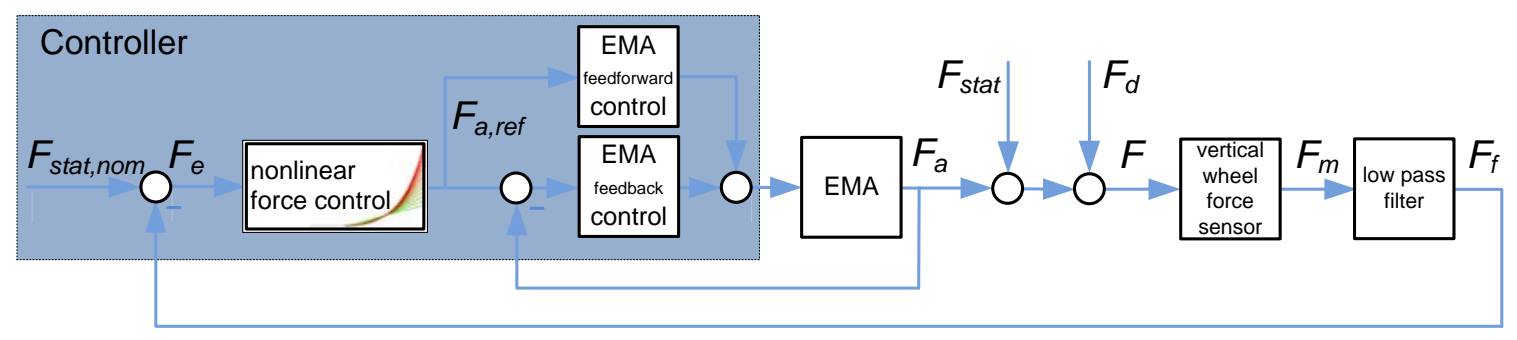

Figure 9: Block diagram of attraction force control using an electromagnetic actuator (EMA) for crosswind stability augmentation of railway vehicles

Figure 9 shows the block diagram of the total system associated with one wheel of the coach. The total vertical force $F$ acting on the wheel is the actuator force $F_{a}$ plus the static wheel load $F_{\text {stat }}$ plus a disturbance force $F_{d}$. The latter comprises the effects of crosswind, of track irregularities and of unmodeled dynamics like the coach rolling motion, carbody dynamics, etc. For simplicity, from the portions of $F_{d}$ only the crosswind term will be considered in the sequel. Nevertheless, the other portions will definitly need to be considered in a final control design. The measured vertical wheel force $F_{m}$ is filtered to remove high frequency system and measurement noise. For the initial design here, we assume a second order Butterworth filter with a bandwidth of $2 \mathrm{~Hz}$. The filtered vertical force $F_{f}$ is considered the controlled variable. All forces are defined with positive sign in downward direction.

The nonlinear force controller computes the actuator set point $F_{a \text {,ref }}$ according to a progressive characteristics depending on the control error $F_{e}$ which is defined as the deviation of $F_{f}$ from the static wheel force.

According to the demands of the EN14067-6, a so-called lay-out case is defined representing the situation when the total vertical wheel rail force takes on the minimum allowed value being $10 \%$ of the static load. Assuming steady state conditions the necessary actuator force $F_{a}^{*}$ in the lay-out case can be derived from:

$$
F_{a}^{*}+F_{\text {stat }, \text { nom }}+F_{d, \max }=F=0.1 \cdot F_{\text {stat }, \text { nom }} \quad \Rightarrow \quad F_{a}^{*}=-0.9 \cdot F_{\text {stat,nom }}-F_{d, \max } \text {. }
$$



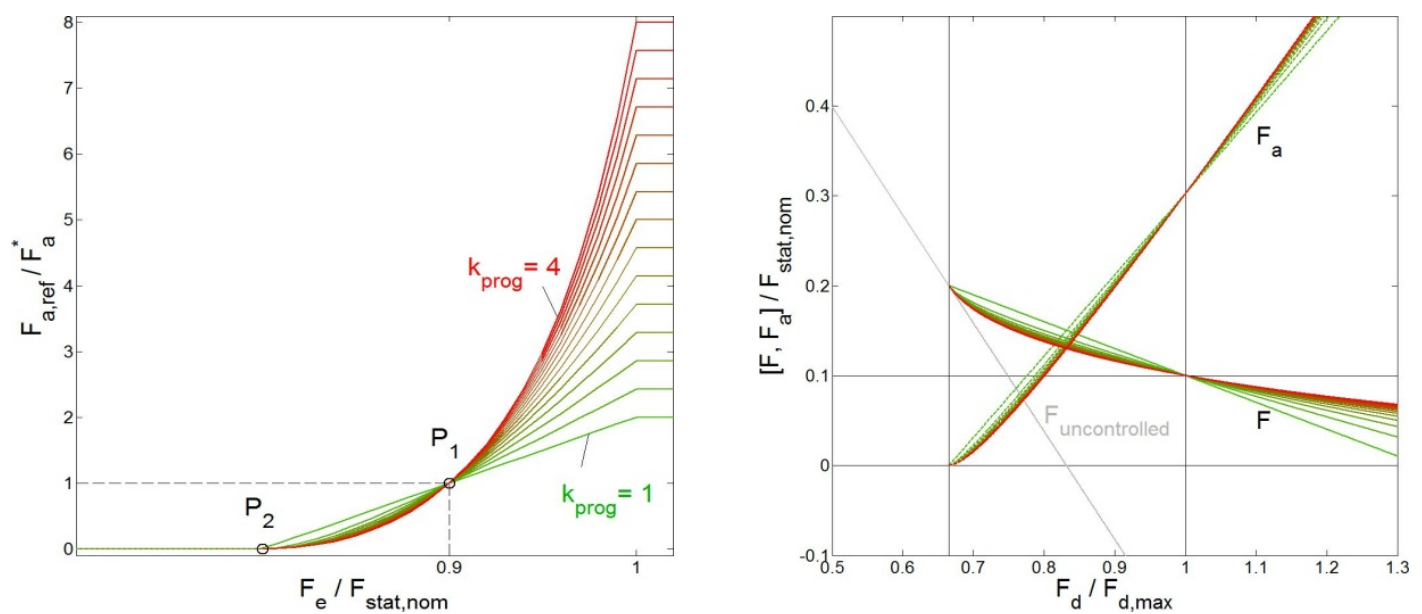

Figure 10: Parameterizable nonlinear control force characteristics (left) and resulting behaviour of the controlled system (right).

Here, the succeeding assumptions were made:

$\mathrm{F}_{\mathrm{d}}=\mathrm{F}_{\mathrm{d}, \max }$

$F_{\text {stat }}=F_{\text {stat nom }}$,

i.e. the cross wind induced lift force takes up its maximum assumed value (lay-out case);

$F=F_{1}=0.1 \cdot F_{\text {stat }, \text { nom }}$,

$F_{f}=F$,

i.e. the nominal static force used in the control is correct;

$F_{a}=F_{a}^{*}=F_{a, r e f}$,

i.e. ideal wheel force sensor;

i.e. the ideal actuator force associated to the lay-out case.

The characteristics of the nonlinear force controller is illustrated in Figure 10 where the control error $F_{e}$ is normalized with the nominal static wheel load $F_{\text {stat,nom }}$ and the actuator setpoint $F_{a, \text { ref }}$ is normalized with $F_{a}^{*}$. The fixed point $P_{1}$ is associated to the lay-out case according to the previous consideration. A second fixed point $P_{2}$ can be used for parameterization such that there is no control action at smaller values of the control error $F_{e}$ than $F_{2}$, i.e. $F_{a, r e f}\left(F_{e}<F_{2}\right)=0$. Thus, unneeded activation of the actuator is avoided in case of high frequency disturbance input with moderate amplitude which may be caused by track irregularities. At $P_{2}$, both $F_{a, r e f}$ and the slope of the characterstics are zero to induce smooth control onset. The total characteristics is representated by

$$
F_{a, r e f}\left(F_{e}\right)=\left\{\begin{array}{cl}
0, & \text { if } F_{e}<F_{2}, \\
\frac{a}{F_{e}-c}+b \cdot F_{e}+d, & \text { if } F_{2} \leq F_{e} \leq F_{\text {stat }, \text { nom }}, \\
F_{a, \text { max }}, & \text { if } F_{e}>F_{\text {stat }, \text { nom }} .
\end{array}\right.
$$

The coefficients $a, b, c, d$ are chosen such that the conditions $P_{1}, P_{2}$ are met. Furthermore, a progression parameter $k_{\text {prog }}$ is introduced and can be tuned to establish a desired nonlinear behaviour as shown in Figure 10. Concisely, the coefficients can be calculated using the following formulae:

$$
\begin{aligned}
& F_{a, \max }=k_{\text {prog }} \cdot F_{a}^{*} \cdot F_{2} /\left(F_{2}-F_{1}\right), \\
& c=F_{1} /\left(1-F_{a, \max } / F_{a}^{*} \cdot\left(1-F_{1} / F_{2}\right)^{2}\right), \\
& b=F_{a}^{*} \cdot\left(F_{1}-c\right) /\left(F_{2}-F_{1}\right)^{2}, \\
& a=b \cdot\left(c-F_{2}\right)^{2}, \\
& d=b \cdot\left(c-2 \cdot F_{2}\right) .
\end{aligned}
$$

Note that in the limit case $k_{\text {prog }}=1$, the characteristics beyond $P_{2}$ degenerates and becomes a straight line, so that the smooth control onset at $P_{2}$ is not given.

In simulations, we were able to in principle demonstrate the effectiveness of the control scheme, cf. Section 4.4. A real control design will have to take both the roll dynamics of the coach chassis and a realistic dynamic model of the EMA into account. The latter needs to cover essential nonlinearitites like saturation and hysteresis, because they will have significant influence on the stability of the controlled system. Such a model is not available at present time for not even a physical actuator exists to our knowledge. Therefore, here are only some qualitative applicational comments for a final control design. Secondary feedforward and/or feedback actuator dynamics compensation may be beneficial to improve its behaviour in terms of increased bandwidth and nonlinearity mitigation in the operating domain. Appropriate low pass filtering of the measured vertical wheel force needs to be designed in order to reduce high frequency disturbances (e.g. from track irregularities and measurement noise) against the rollover critical low frequency content as induced by crosswind. 
Next, the nonlinear characteristics is to be parameterized for sufficient performance on the basis of simulating critical scenarios. Linear control design methods can be used to determine the maximum slope of the characteristics. Finally, the absolute stability of the total nonlinear system can be proven using the Popov stability criterion [25] for sector nonlinearities.

\subsection{Multibody Simulation}

The concept was additionally examined using an elaborate multibody simulation in SIMPACK 9.3. The simulation scenario here considered transient wind loads due to the wind gust model in Figure 11 that were applied to the complete train set and complies with the definitions in Section 5.4.4 of the EN 14067-6 [3]. Hence, this scenario could be used for homologation of a high speed train according to the TSI [2]. In comparison, the simulations presented in Section 2 of this paper accords with Section 5.4.3 of EN 14067-6. They are well suited for surveys in an early design phase of the vehicle, since only quasi-static wind loads applied to the so-called five-mass-model of a single car are taken into account.

The SIMPACK vehicle model of the NGT consists of the train head, four intermediate coaches and the trailing train head. The vehicles have two level suspensions and independently rotating wheels with mechatronic track guidance. In order to ensure a good ride comfort, active lateral centering devices in the secondary suspension minimize the use of the lateral bumpstops. The vehicle model and the controller for the mechatronic track guidance are described in [26]. The aerodynamic coefficients for the vehicles are identical to those used in Section 2 and originate from wind The train runs at $400 \mathrm{~km} / \mathrm{h}$ on a straight track on flat ground. The transient wind load corresponds to the so-called Chinese hat in EN 14067-6 which is an equivalent deterministic gust representation to approximate turbulent wind that actually is a random process. Although Baker et al [1] favor a stochastic approach "to correctly reproduce the interaction between vehicle dynamics and turbulent wind", the Chinese hat representation seems to be sufficient to evaluate significant answers in the context of this potential study since it is even accepted for homologation purposes. The wind gust in Figure 11 features a characteristic frequency of 0.0425 $\mathrm{Hz}$ and is assumed to be spatially fixed, i.e. each track length coordinate $s$ is associated to one wind velocity $v$, i.e. $v=v(s)$ in Figure 11. A maximum wind speed at the gust of $26.8 \mathrm{~m} / \mathrm{s}(96.5 \mathrm{~km} / \mathrm{h})$ is assumed extrapolating the CRWC defined by the TSI [2], see Figure 3. The wind direction with respect to the track is $90^{\circ}$. The scenario starts with an increasing wind up to a steady wind speed of $15.8 \mathrm{~m} / \mathrm{s}(56.9 \mathrm{~km} / \mathrm{h})$. The resulting wind force and torque applied to each carbody is evaluated using the sliding mean value of the wind velocity with respect to the carbody length.tunnel measurements performed at Reynolds Number of $0.75 \cdot 10^{6}$.

In the simulation, the vertical wheel forces were directly obtained from the wheel/rail contact module of SIMPACK. These measured vertical forces were low pass filtered as presented in Section 4.3,The actuator-force is calculated with the proposed force law, in which the parameters $k_{\text {prog }}=2.5, F_{d, \max }=0.92 F_{\text {stat,nom }}$ and $F_{2}=0.8 F_{\text {stat,nom }}$ were introduced.

The results of the simulations are summarized in Figure 12. The plots in the first row show, how the low pass filtered vertical wheel forces $F_{f}$ at the right hand side of the vehicle change along the whole simulation distance, but no Attraction Control is considered, i.e. $F_{a}=0$. As prescribed by [3], a fourth order Butterworth filter with $2 \mathrm{~Hz}$ cut-off frequency has been used for this assessment of $F_{f}$. In each diagram, the red curve presents the force of the leading and the blue one the force of the trailling wheel as a function of $s$, i.e. $F_{f}=F_{f}(s)$. The vehicle starts with nearly nominal wheel load only slightly excitated by track irregularities. The steady crosswind causes a first

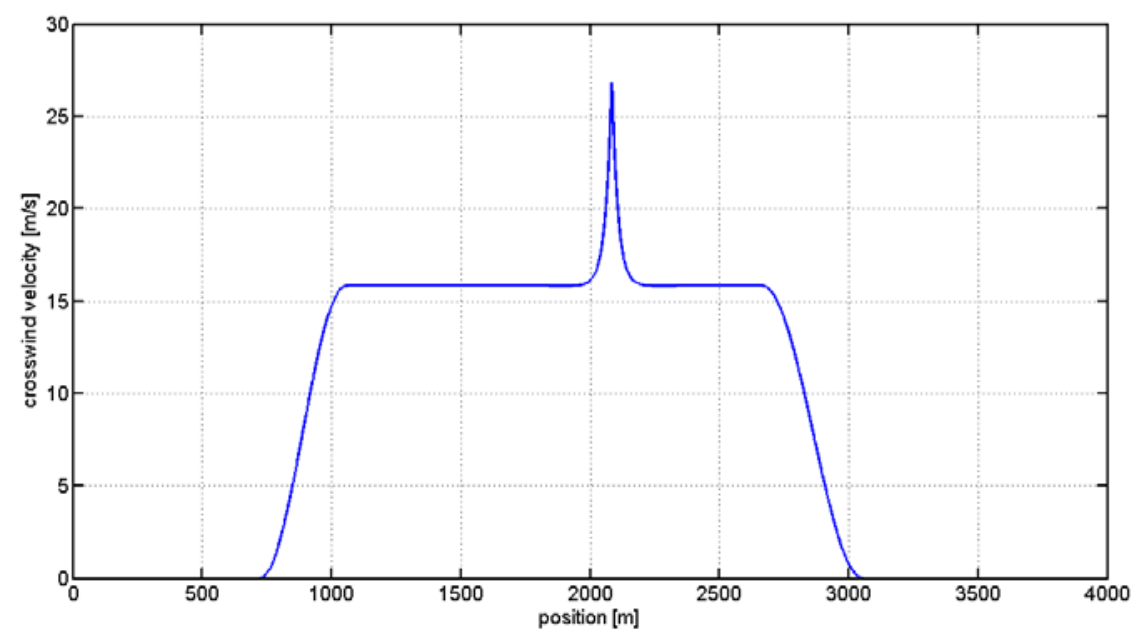

Figure 11: Crosswind velocity definition "Chinese Hat" according to EN 14067-6 [3] 
wheel unloading. The wind gust acts on the vehicle at a running distance between 2.0 and $2.2 \mathrm{~km}$ and results in an unacceptable wheel unloading at the intermediate coach. The wheel force temporarily becomes less than the limit value of $0.1 F_{\text {stat,nom }}$ indicated by the black dash-dotted line. The second row of the diagrams in Figure 12 zooms in to show the intervall of the gust in more detail. It can be seen that the leading wheel of the coach is transitorily unloaded, i.e. $F_{f}\left(s=s_{u}\right)=0$. The trailing train head that only differs from the leading train head in its orientation with respect to the flow field exposes an uncritical unloading behaviour.

The plots in the third row of Figure 12 delineate the results for the second simulation scenario, now with Attraction Control applied. Due to the attraction forces induced by the EMAs that are given in the last row of Figure 12, the vertical wheel force now remains above the limit value for all wheels.
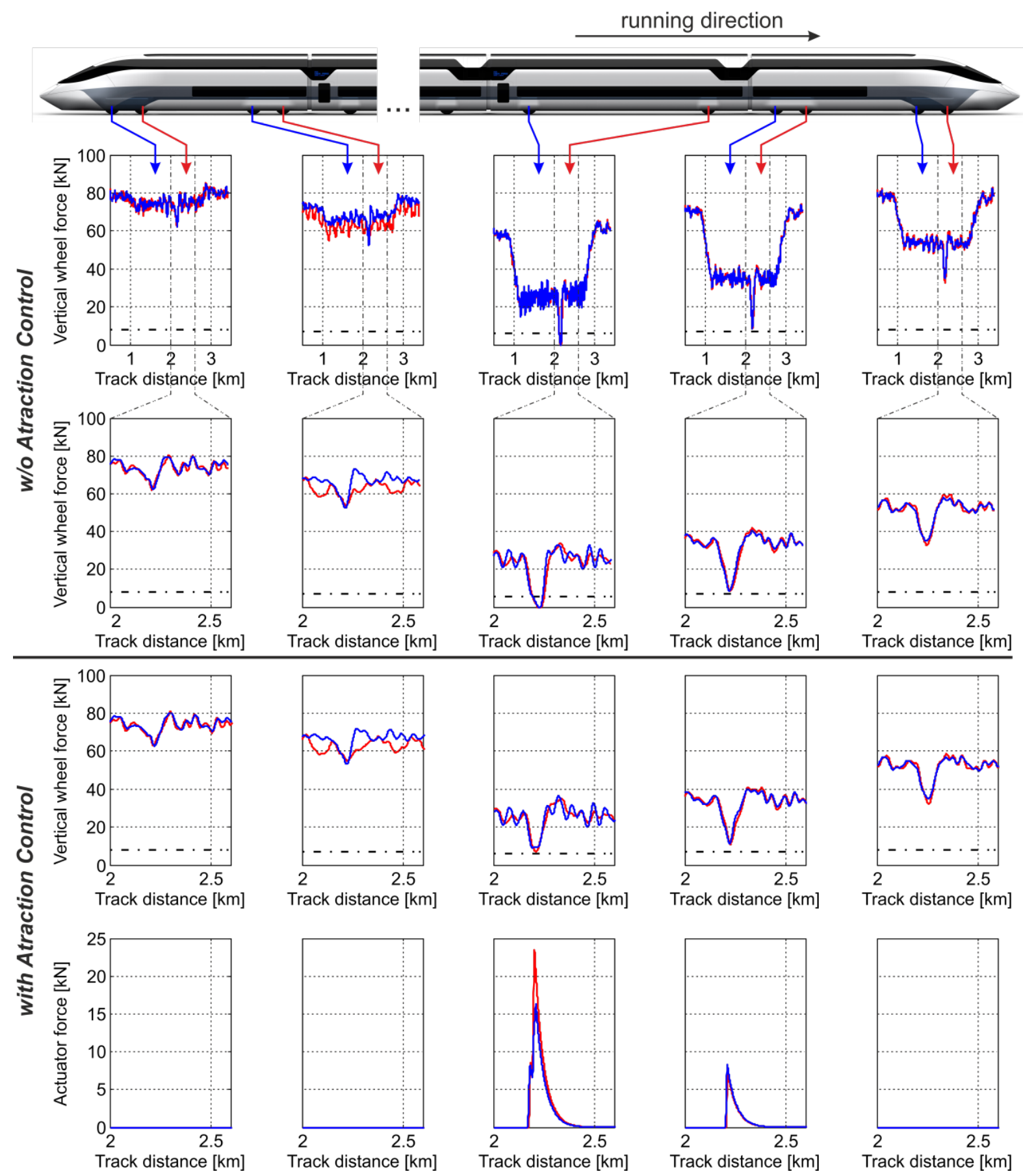

Figure 12: Vertical wheel rail forces at the windward side for both crosswind scenarios: w/o Attraction Control (top) and with Attraction Control (bottom) 
At the second running gear of the leading train head (plot in the last row, 4th column), the EMA also applies an atraction force although the wheel unloading has acceptable values in the scenario without control. This is a result of the nonlinear force law from Section 4.3 with a smooth actuator onset at $P_{2}$, see Figure 10, which requires the actuator to start-up a pre-defined step before the actual limit value of $0.1 F_{\text {stat,nom }}$ is touched. Due to the choice of $F_{2}=0.8 F_{\text {stat,nom }}$ in the simulation scenario, the actuator is engaged for every force smaller than $0.2 F_{\text {stat,nom }}$. Besides, the used parameters of the force law given above were tuned in such a way, that the actuator forces in the simulated scenario do not exceed $25 \mathrm{kN}$ and the wheel unloading is in an acceptable range at all wheels.

The maximum actuator force here reached values up to $23.5 \mathrm{kN}$, while in the simplified quasi-static scenario in Section 3.4, $39 \mathrm{kN}$ were needed to ensure crosswind stability, Table 1. So the results of the elaborate transient multibody simulation seem to alleviate the demands on the required actuator force, although such a statement should actually be founded on more than one or two simulations considering different angles of attack and unbalanced lateral accelerations. On the other hand, it is a reasonable assumption that simplified scenarios facilitate the survey on the stability of vehicle concepts but have to consider higher safety margins in order to somehow take disregarded aspects into account that are associated with the simpification. In any case, the rating that the lightweight intermediate coaches of the NGT are the central matter of concern with respect to crosswind stability can congruently be concluded from both simulation scenarios.

\section{CONCLUSIONS AND OUTLOOK}

The present paper discusses the feasibility of different approaches to ensure crosswind stability by means of active control using the example of DLR's Next Generation Train. The Attraction Control approach turned out to be the most promising one. Therefore this concept was examined in more details. The simulation results provide confidence that Attraction Control in principle is a feasible approach. However, crosswind stability is highly safety relevant. Therefore high demands on active control devices are to be made and objective evidence on its reliability is to be provided. Hence, this fact reduces the chances for realization of such a concept in the short run. In addition, the effects of Attraction Control on the infrastructure, this applies to the pulling-out forces acting on the rail and the electromechanical compatibility of the actuator, are an open issue.

Nevertheless the presented results motivate further research activities in order to allow for lightweight design of very high speed trains in the future. The design of an electromagnetic actuator needs to be tailored for crosswind stability application as mentioned in Section 4.1. The sensor technology will be further developed not only in this context but as well as related to the mechatronic guidance concept of the running gears of the NGT. More efforts need to be invested to further examine the suitability of the proposed Attraction Control set-up for a more complete range of real life scenarios. Curved track including transitions, turbulent crosswind presented as a stochastic process, parametric uncertainties e.g. regarding to the aerodynamic coefficients, line configurations like embankments or viaducts are issues to be considered in detail. Last but not least new research facilities at DLR will help to advance the fundamental knowledge on the flow field under the influence of high crosswinds. The expected outcome is a better specification of the transient aerodynamic forces that facilitates less conservative homologation procedures and inspires new ideas for aerodynamically beneficial shapes of the outer vehicle contour. In summary, crosswind stability will continue to play a prominent role within the Next Generation Train project of DLR.

\section{Acknowledgements}

Some ideas of the paper were introduced into DLR's Next Generation Train project by Dr. Antonio Carrarini during his period of employment at DLR. This in particular applies to ideas, statements and literature research results in Section 3.1 and Section 3.2 [10].

Apart from the general DLR funding this work was supported by BMBF (BMBF Förderkennzeichen: 01IS12022G), the German Federal Ministry of the Education and Research, within the ITEA 2 project Modrio.

\section{References}

[1] C. Baker, F. Cheli, A. Orellano, N. Paradot, C. Proppe and D. Rocchi, "Cross-wind effects on road and rail vehicles," Vehicle System Dynamics, vol. 47, no. 8, pp. 983-1022, 2009.

[2] TSI HS RST: Technical Specification for Interoperability relating to the Rolling Stock sub-system of the trans-European High-Speed rail system, 2008.

[3] EN 14067-6: 2010 Railway Applications -Aerodynamics- Requirements, 2010.

[4] A. Orellano, T. Schwiegel and R. Kirchhof, "Aerodynamische und thermische Optimierung des ZEFIRO-Hochgeschwindigkeitszuges," ETR. Eisenbahntechnische Rundschau, vol. 61, no. 1-2, pp. 28-33, 2012. 
[5] B. Diedrichs, M. Ekequist, S. Stichel and H. Tengstrand, "Quasi-static modelling of wheel-rail reactions due to crosswind effects for various types of high-speed rolling stock," Proceedings of the Institution of Mechanical Engineers, Part F: Journal of Rail and Rapid Transit, vol. 218, no. 2, pp. 133-148, 2004.

[6] G. Kopp, J. König, J. Winter, M. Fischer and S. Ehrenberger, "Systematic derivation of the NGT rail vehicle concept," RTR Special - Next Generation Train, 2011.

[7] S. Loose and A. Heckmann, "Contributions to aerodynamics," RTR Special - Next Generation Train, 2011.

[8] A. Carrarini, Reliability based analysis of the crosswind stability of railway vehicles, Aachen: Shaker-Verlag, 2006.

[9] A. Orvnäs, S. Stichel and R. Persson, "On-track tests with active lateral secondary suspension: a measure to improve ride comfort," ZEV Rail Glasers Annalen, vol. 132, no. 11-12, pp. 469-477, 2008.

[10] A. Carrarini, Überlegungen zur Regelung der Sekundärfederung von Schienenfahrzeugen bei Seitenwindbelastungen, DLR Internal Report IB 515-09-12, 2009.

[11] M. Hofmann, "Design of a linear induction machine for railway systems using finite element calculation," Shaker, Aachen, 2002.

[12] T. Werle, Bemessung und Vergleich von Linear-Boostern für den Einsatz bei elektrischen Bahnen, Aachen: Shaker, 2003.

[13] B. Funieru, Design and Optimization of a Linear DC Actuator used as Tractive Effort Booster in Railway Applications, Shaker, 2008.

[14] DB Netz AG, Richtlinie 807.04 Bautechnik, Leit-, Signal- und Telekommunikationstechnik. Ausgewählte Maßnahmen und Anforderungen and das Gesamtsystem Fahrweg/Fahrzeug - Aerodynamik/Seitenwind, DB Netz AG, Ed., 2006.

[15] The Modelica Association, "Modelica," [Online]. Available: http://www.modelica.org. [Accessed April 2013].

[16] M. Bocciolone, F. Cheli, R. Corradi, S. Muggiasca and G. Tomasini, "Crosswind action on rail vehicles: Wind tunnel experimental analyses," J. of Wind Eng. and Ind. Aerodynamics, pp. 584-610, 2008.

[17] B. Diedrichs, "On computational fluid dynamics modeling of crosswind effects for high-speed rolling stock," Journal of Rail and Rapid Transit - Proc. Instn Mech. Engrs Part F, pp. 203-226, 2003.

[18] D. Thomas, B. Diedrichs, M. Berg and S. Stichel, "Dynamics of a high-speed rail vehicle negotiating curves at unsteady crosswind," Proceedings of the Institution of Mechanical Engineers, Part F: Journal of Rail and Rapid Transit, pp. 567-579, 2010.

[19] T. Grossenbacher and R. Schneider, "Trials with the test model of the new double-deck car with rolling compensation FLEXX Tronic WAKO of SBB," ZEVrail Glasers Annalen, vol. 135, pp. 108-117, Nov. 2011.

[20] S. Johansson, L. Hakansson, P. Persson and I. Claesson, "Active Control of Lateral Vibrations in a Railway Carriage," International Journal, vol. 7, 2002.

[21] J. Katz, "Aerodynamics of Race Cars," Annual Review of Fluid Mechanics, vol. 38, pp. 27-63, 2006.

[22] S. Loose, A. Lauterbach, S. Algermissen and A. Heckmann, "Activities in acoustics," RTR Special - Next Generation Train, pp. 51-60, 2011.

[23] F. Hörl and D. Leinhos, "Einsatzmöglichkeiten der Linearmotortechnik im Rad-Schiene-System," eb Elektrische Bahnen, vol. 99, no. 10, 2001.

[24] B. Kurzeck and L. Valente, "Design aspects of a mechatronic track guidance,” in 22nd Symposium on Dynamics of Vehicles on Roads and Tracks, Manchester Metropolitan University, UK, 2011.

[25] V. Popov, ”Absolute stability of nonlinear systems of automatic control,” Automation and Remote Control, vol. 22, pp. 857-875, 1962.

[26] B. Kurzeck, A. Heckmann, C. Wesseler and M. Rapp, "Mechatronic track guidance on disturbed track: the trade-off between actuator performance and wheel wear," in 23nd Symposium on Dynamics of Vehicles on Roads and Tracks, Qingdao, China, 2013.

[27] S. Terfloth, "Wikipedia," published under CC SA 3.0. [Online]. Available: http://en.wikipedia.org/wiki/Eddy_current_brake. [Accessed 201304 12]. 